\title{
Impact of massive neutrinos on the Higgs self-coupling and electroweak vacuum stability
}

\author{
Werner Rodejohann ${ }^{1, *}$ and He Zhang ${ }^{1, \oplus}$ \\ ${ }^{1}$ Max-Planck-Institut für Kernphysik, \\ Saupfercheckweg 1, 69117 Heidelberg, Germany
}

\begin{abstract}
The presence of right-handed neutrinos in the type I seesaw mechanism may lead to significant corrections to the RG evolution of the Higgs self-coupling. Compared to the Standard Model case, the Higgs mass window can become narrower, and the cutoff scale become lower. Naively, these effects decrease with decreasing right-handed neutrino mass. However, we point out that the unknown Dirac Yukawa matrix may impact the vacuum stability constraints even in the low scale seesaw case not far away from the electroweak scale, hence much below the canonical seesaw scale of $10^{15} \mathrm{GeV}$. This includes situations in which production of right-handed neutrinos at colliders is possible. We illustrate this within a particular parametrization of the Dirac Yukawas and with explicit low scale seesaw models. We also note the effect of massive neutrinos on the top quark Yukawa coupling, whose high energy value can be increased with respect to the Standard Model case.
\end{abstract}

*Electronic address: werner.rodejohann@mpi-hd.mpg.de

$\dagger$ Electronic address: he.zhang@mpi-hd.mpg.de 


\section{INTRODUCTION}

Direct observational constraints on the Higgs boson [1-3] suggest that, if it exists, its detection should be close. Apart from direct limits, indirect limits from electroweak precision data have been obtained [4]. Another approach to study Higgs properties, on which we focus here, analyzes the high energy values of the Higgs self-coupling [5] 7].

Within the framework of the Standard Model, the one-loop renormalization group equation (RGE) of the Higgs self-coupling $\lambda$ is given by $(4 \pi)^{2} \frac{\mathrm{d} \lambda}{\mathrm{d} \ln \mu}=\beta_{\lambda}$, with [8]

$$
\begin{gathered}
\beta_{\lambda}=6 \lambda^{2}-\lambda\left(3 g_{1}^{2}+9 g_{2}^{2}\right)+\left(\frac{3}{2} g_{1}^{4}+3 g_{1}^{2} g_{2}^{2}+\frac{9}{2} g_{2}^{4}\right) \\
+4 \lambda \operatorname{tr}\left(3 Y_{u}^{\dagger} Y_{u}+3 Y_{d}^{\dagger} Y_{d}+Y_{\ell}^{\dagger} Y_{\ell}\right)-8 \operatorname{tr}\left[3\left(Y_{u}^{\dagger} Y_{u}\right)^{2}+3\left(Y_{d}^{\dagger} Y_{d}\right)^{2}+\left(Y_{\ell}^{\dagger} Y_{\ell}\right)^{2}\right]
\end{gathered}
$$

Here $g_{i}$ are the gauge couplings and $Y_{f}$ (for $f=u, d, \ell$ ) denote Yukawa coupling matrices of up-quarks, down-quarks and charged leptons. Two important limits exist when one runs $\lambda$ from the weak scale to larger scales:

- in the limit of small $\lambda$, the top quark Yukawa drives $\lambda$ to negative values, and the Higgs potential is no longer bounded from below. This leads to the vacuum stability bound;

- in the limit of large $\lambda$, the Higgs self-coupling drives $\lambda$ to even larger values, and the presence of a non-perturbative coupling leads to the triviality bound.

These two aspects imply lower and upper values of the Higgs mass, depending on the embedding (or cutoff) scale $\Lambda$ of the theory. In particular, for interesting values of Higgs mass around $125 \pm 2 \mathrm{GeV}$, latest numerical analyses in the literature [9 11] indicate that $\Lambda$ should be around $10^{9} \sim 10^{11} \mathrm{GeV}$ including uncertainties from the top quark mass, the strong coupling and higher order effects. Slightly larger Higgs masses would cause no conceptual problems up to the Planck scale, even larger values would lead to non-perturbative $\lambda$ before the Planck scale.

The above consequences can be significantly altered if the $\beta$-function of $\lambda$ is modified. Necessarily, physics beyond the Standard Model needs to be added. There is already a wellestablished field of new physics: massive neutrinos. We therefore analyze the possibility that the physics behind neutrino mass influences the theoretical Higgs constraints.

The most straightforward mechanism to generate small active neutrino masses is the type I seesaw mechanism [12], in which case neutrino masses are given by

$$
m_{\nu}=\frac{m_{D}^{2}}{M_{R}}=Y_{\nu}^{2} \frac{v^{2}}{M_{R}}
$$


with $Y_{\nu}=m_{D} / v$ the Yukawa coupling of the neutrinos, $v$ the Standard Model vacuum expectation value and $M_{R}$ the mass scale of heavy right-handed neutrinos. A Yukawa coupling of order one, and a neutrino mass scale of 0.05 to $0.1 \mathrm{eV}$, implies for $M_{R}$ roughly $10^{14}$ to $10^{15} \mathrm{GeV}$, which defines its canonical scale. Naively, decreasing $M_{R}$ indicates smaller Yukawa couplings. If we run now $\lambda$ from low to high scale, and cross the threshold at which the heavy neutrinos are integrated out, the Yukawa couplings of the neutrinos generate the following contribution to the $\beta$-function of the Higgs self-coupling [13]:

$$
\Delta \beta_{\lambda}=4 \lambda \operatorname{tr}\left(Y_{\nu}^{\dagger} Y_{\nu}\right)-8 \operatorname{tr}\left[\left(Y_{\nu}^{\dagger} Y_{\nu}\right)^{2}\right]
$$

We see that the effect of the Dirac Yukawas is very similar to the one of the top quark, and thus will influence the vacuum stability bound. One expects that the Higgs mass window will become narrower, and that the cutoff scale of the theory will decrease [10, 14 17]. Sizable effects on $\lambda$ imply sizable Yukawa couplings $Y_{\nu}$, and thus heavy neutrino masses around $10^{13} \sim 10^{15} \mathrm{GeV}$. Lighter right-handed neutrinos reduce the Yukawas and correspondingly their effect on Higgs mass constraints.

However, in this paper we note that even low scale seesaw models can have an impact. The simple reason is that the seesaw formula contains matrices instead of numbers, and thus the argumentation from above can be avoided: large Yukawa couplings can work very well with low scale right-handed neutrinos, even going down to the TeV scale. Note that the scale of the right-handed singlets is a priori a free and unknown parameter, and might not correspond to the "canonical" scale of $\gtrsim 10^{14} \mathrm{GeV}$. While this superheavy scale is expected from the point of view of naturalness, one should note that also a vanishing right-handed neutrino mass is natural, in the sense that the symmetry of the Lagrangian is enhanced in that case. Hence, the scale of $M_{R}$ is unknown and might be at the phenomenologically interesting $\mathrm{TeV}$ scale. In order to have sizable mixing of $\mathrm{TeV}$ singlets with the SM particles, some tuning is necessary, which can however be arranged in models. It requires some peculiar flavor structure in the matrices, and one notes that the unexpected mixing scheme of leptons seems to hint towards rather non-trivial flavor structure. This is a framework often applied in the literature [18 20], and we will discuss the impact on the Higgs mass bounds in this work.

In this situation described above, the $\beta$-function of $\lambda$ receives sizable corrections even at low energy scales, with implications on the possible cutoff scale. We will give here examples on the consequences of (in particular low scale) type I seesaw for the evolution of the Higgs self-coupling by using a general parametrization of the unknown Dirac Yukawas, as well as by applying two explicit models realizing low scale seesaw with large Yukawas. We note that sizable effects of neutrino Yukawas on the Higgs self-coupling can be expected in situations in which production of right-handed neutrinos at colliders is possible, because this can only be achieved if the Yukawas are large enough. 
The remaining parts of this work are organized as follows: In Sec. II we present the framework of type I seesaw and its influence on the evolution of the Higgs self-coupling. Numerical analyses illustrating this will be performed in Sec. III, where we also discuss the impact on the top Yukawa coupling. Finally, in Sec. IV] we summarize our results and conclude.

\section{SEESAW IMPACT ON $\beta_{\lambda}$}

Among various attempts to extend the Standard Model (SM) in order to accommodate massive neutrinos, the type I seesaw mechanism turns out to be a very attractive one, in view of its natural and elegant explanation of light neutrino mass scales. Before studying

the vacuum stability in the seesaw model, we first briefly review the type I seesaw and the parametrization utilized throughout the remaining parts of this work.

\section{A. Right-handed neutrinos and seesaw}

In order to generate light neutrino masses, one typically introduces three right-handed neutrinos besides the Standard Model particle content. The corresponding Lagrangian reads

$$
-\mathcal{L}_{\nu}=\overline{\nu_{R}} Y_{\nu} \ell_{L} \tilde{H}^{\dagger}+\frac{1}{2} \overline{\nu_{R}} M_{R} \nu_{R}^{c}+\text { h.c. }
$$

where $\tilde{H}=\mathrm{i} \tau_{2} H^{*}$ is the Higgs doublet, and $Y_{\nu}$ denotes the Yukawa coupling matrix. At energy scales below the lightest right-handed neutrino threshold, the light neutrino mass matrix is given by the well-known seesaw formula [12]

$$
m_{\nu}=v^{2} Y_{\nu}^{T} M_{R}^{-1} Y_{\nu}
$$

where $v$ is the vacuum expectation value of $H$. Ignoring the flavor structures, Eq. (5) allows us to naively estimate the magnitude of $Y_{\nu}$. For example, for $m_{\nu}=\mathcal{O}(0.1) \mathrm{eV}$, one has $Y_{\nu}$ of order unity if $M_{R}=\mathcal{O}\left(10^{14}\right) \mathrm{GeV}$. In what follows, we shall consider however the flavor structure of $Y_{\nu}$ by using an explicit parametrization scheme.

Adopting the convention defined in Ref. [21], we make use of the following parametrization of $Y_{\nu}$,

$$
Y_{\nu}=\frac{1}{v} \sqrt{\bar{M}_{R}} R \sqrt{\bar{m}_{\nu}} U^{\dagger}
$$

where $\bar{M}_{R}$ and $\bar{m}_{\nu}$ stand for the diagonal mass matrices of heavy and light neutrinos, respectively. Here $U$ is a unitary (PMNS) matrix diagonalizing $m_{\nu}$ as $\bar{m}_{\nu}=U^{T} m_{\nu} U \equiv$ $\operatorname{diag}\left(m_{1}, m_{2}, m_{3}\right)$ with $m_{i}$ being light neutrino masses. $R$ denotes a complex orthogonal 
matrix which we parameterize as

$$
R=O e^{\mathrm{i} A}
$$

where both $O$ and $A$ are real matrices. A similar parametrization has been discussed in a different context in [22]. The orthogonality of $R$ implies that $O$ is orthogonal and $A$ is antisymmetric, i.e.,

$$
A=\left(\begin{array}{ccc}
0 & a & b \\
-a & 0 & c \\
-b & -c & 0
\end{array}\right)
$$

with real $a, b, c$. We will show in the following section that such a parametrization scheme is particularly useful in discussing the evolution of $\lambda$.

\section{B. Corrections to the RG evolution of $\lambda$}

At energy scales above the right-handed neutrino threshold additional contributions to the SM $\beta$-functions have to be considered in the evolution of all physical parameters. For the Higgs self-coupling, $\beta_{\lambda}$ receives corrections repeated here for convenience [13]:

$$
\Delta \beta_{\lambda}=4 \lambda \operatorname{tr}\left(Y_{\nu}^{\dagger} Y_{\nu}\right)-8 \operatorname{tr}\left[\left(Y_{\nu}^{\dagger} Y_{\nu}\right)^{2}\right]
$$

at one-loop level. The first term turns to be small when $\lambda$ approximates to zero, whereas the second term is not suppressed by $\lambda$ and may affect the RG evolution of $\lambda$ significantly for large $Y_{\nu}$. In general, the presence of right-handed neutrinos drives $\lambda$ towards smaller values at higher energy scales.

For simplicity, we assume that the right-handed neutrino masses are degenerate, i.e., $M_{1}=M_{2}=M_{3}=M_{0}$, which allows us to match the effective theory onto the full theory at a unified scale $\mu=M_{0}$. Note that only the combination $Y_{\nu}^{\dagger} Y_{\nu}$ enters the $\beta$-function of $\lambda$, and one can easily prove that $\operatorname{tr}\left(Y_{\nu}^{\dagger} Y_{\nu}\right)$ depends only on the real parameters in $A$ :

$$
\begin{aligned}
\operatorname{tr}\left(Y_{\nu}^{\dagger} Y_{\nu}\right) & =\operatorname{tr}\left[\frac{M_{0}}{v^{2}} U \sqrt{\bar{m}_{\nu}}\left(e^{\mathrm{i} A}\right)^{\dagger} e^{\mathrm{i} A} \sqrt{\bar{m}_{\nu}} U^{\dagger}\right]=\frac{M_{0}}{v^{2}} \operatorname{tr}\left[\sqrt{\bar{m}_{\nu}} e^{2 \mathrm{i} A} \sqrt{\bar{m}_{\nu}}\right] \\
& =\frac{M_{0}}{v^{2}}\left[\sum_{i} m_{i}+\frac{4(\cosh r-1)}{r^{2}}\left(m_{1}\left(a^{2}+b^{2}\right)+m_{2}\left(a^{2}+c^{2}\right)+m_{3}\left(b^{2}+c^{2}\right)\right)\right]
\end{aligned}
$$

where $r=2 \sqrt{a^{2}+b^{2}+c^{2}}$. One observes that neither the rotation matrix $O$ nor the mixing matrix $U$ affects the evolution of $\lambda$ directly. Furthermore, the hyperbolic cosine factor involving $r$ plays a crucial role in seesaw models since it could enhance the magnitude of $Y_{\nu}$ without spoiling the values of the light neutrino masses. In other words, the $R$ matrix 
elements can be arbitrarily large in virtue of the fact that $R$ disappears from the seesaw formula playing no role in determining the light neutrino masses ${ }^{1}$. However, $R$ does influence the Yukawa couplings in $Y_{\nu}$ which could lead to sizable impact on the running of $\lambda$.

In case that the light neutrino masses are quasi-degenerate, namely $m_{1} \simeq m_{2} \simeq m_{3}=m_{0}$, Eq. (10) can further be simplified to

$$
\operatorname{tr}\left(Y_{\nu}^{\dagger} Y_{\nu}\right) \simeq \frac{M_{0} m_{0}}{v^{2}}(1+2 \cosh r) .
$$

As a numerical example, we take $M_{0}=1 \mathrm{TeV}, m_{0}=0.1 \mathrm{eV}, r=25$, and obtain $\operatorname{tr}\left(Y_{\nu}^{\dagger} Y_{\nu}\right)=$ $\mathcal{O}(0.1)$. The naive estimate from $m_{\nu}=Y_{\nu}^{2} v^{2} / M_{R}$ would give $Y_{\nu}^{2} \sim 10^{-11}$ and thus a negligible effect. Therefore, even for very light right-handed neutrinos, sizable Yukawa couplings are still acceptable when the matrix structure of $Y_{\nu}$ is taken properly into account. Arranging sizable Dirac Yukawas in case of a low seesaw scale is often done in order to construct scenarios in which the seesaw messengers are to be produced at colliders. The reason is that the mixing with the SM particles is given by $S \sim Y_{\nu} v / M_{R}$, which needs to be not too small in order to allow sizable cross sections [23 25]. As a further illustration of this, consider the case in which the mixing of one right-handed neutrino with SM particles, say, electrons, saturates the present bound [26]: $\left|S_{e i}\right|^{2} \leq 0.0054$. This implies that $Y_{\nu} \simeq 0.4\left(M_{R} / \mathrm{TeV}\right)$. We stress that if right-handed neutrinos can be produced at colliders, the effects on the Higgs self-coupling and electroweak vacuum stability that we discuss in this paper can be expected.

We note that the above analytical approximations rely on the assumption of a degenerate (or nearly degenerate) right-handed neutrino mass spectrum. Relaxing this assumption will not allow us to eliminate the rotation matrix $O$ in Eq. (10), and thus increase the number of free parameters (three real rotation angles are necessary to parameterize $O$ ). It will furthermore complicate the analysis significantly, as several thresholds would have to be taken into account. In analogy to Refs. [10, 14, 17], we will stick to degenerate heavy neutrinos, but in contrast to those works (which used $R=\mathbb{1}$ ) take the flavor structure of the Yukawa matrix into account.

Finally we stress that the complexity of $e^{\mathrm{i} A}$ is crucial in the leptogenesis mechanism [27]. The presence of light right-handed neutrinos may also have impact on various low scale phenomena, e.g., lepton flavor violating processes, non-unitarity effects in neutrino oscillations, neutrinoless double beta decay as well as signatures at colliders.

\footnotetext{
${ }^{1}$ Obviously, the elements of $Y_{\nu}$ need to be perturbative.
} 


\section{NUMERICAL ILLUSTRATIONS}

We proceed to illustrate the previous discussion on how the right-handed neutrinos affect the evolution of $\lambda$. In our numerical analysis, we make use of the input physical parameters (e.g., fermion masses, gauge couplings, flavor mixing parameters) from recent re-evaluations of running SM parameters normalized at the electroweak scale, i.e., $\mu=M_{Z}$ [11, 28]. The full set of two-loop RGE [29] for $\lambda$ is solved together with the one-loop matching condition for $\lambda(\mu)$ and $m_{t}$ [30]. For simplicity, we also assume $a=b=c=a_{0}$, implying that $r=2 \sqrt{3} a_{0}$. In addition, only the normal mass ordering $\left(m_{1}<m_{2}<m_{3}\right)$ of light neutrinos is considered; for a nearly degenerate neutrino mass spectrum there is no quantitative difference between normal and inverted mass orderings. For simplicity, and in order to focus on the impact of the neutrino sector, we will use the top quark pole mass $m_{t}^{\text {pole }}=172.9 \mathrm{GeV}$ advocated by the Particle Data Group [31], and will not take into account the effect of varying the top quark mass or the strong coupling.

\section{A. Corrections to the Higgs self-coupling}

In Fig. 1 we show the impact of right-handed neutrinos on the evolution of $\lambda(\mu)$. For illustration purpose, we take $m_{H}=125 \mathrm{GeV}$ and $m_{H}=135 \mathrm{GeV}$ together with the light neutrino mass $m_{1}=0.1 \mathrm{eV}$. The value $125 \mathrm{GeV}$ corresponds to a situation in which even in the SM there is a non-trivial cutoff scale, whereas $135 \mathrm{GeV}$ is for our parameter choice a value which in the absence of SM extensions causes no conceptual issues up to the Planck scale. The red curves in Fig. 1 show the running behavior in the pure SM case, from which one can see that for $m_{H}=125 \mathrm{GeV}$ the self-coupling $\lambda$ crosses zero at energy scales around $10^{10} \mathrm{GeV}$, indicating an upper bound on the potential new physics scale. In case of $m_{H}=135 \mathrm{GeV}$, the $\mathrm{SM}$ is valid in principle up to the Planck scale. The green and blue curves show the running of $\lambda$ with right-handed neutrino mass scale $M_{0}=10^{11} \mathrm{GeV}$ and $M_{0}=10 \mathrm{TeV}$, respectively. For the left plots, we switch off the $A$ matrix by setting $a_{0}=0$. As can be seen from the plot, the evolution of $\lambda$ is barely affected by right-handed neutrinos (the curves almost overlap with each other), which is consistent with our analytical analysis since $Y_{\nu}$ is small in order to compensate a light right-handed neutrino scale. However, when we switch on the real parameters in the $A$ matrix, the evolution of $\lambda$ is modified significantly after crossing the seesaw threshold. Even for relatively light right-handed neutrinos, one can see clearly the shift of the cutoff scale down to low energy ranges. Noteworthy is for instance that the case $m_{H}=135 \mathrm{GeV}$ (which is safe in the SM) can have an unstable vacuum. This can happen even in the canonical case without any non-trivial $R$, as can be seen by the green line corresponding to $M_{0}=10^{14} \mathrm{GeV}[14]$. 

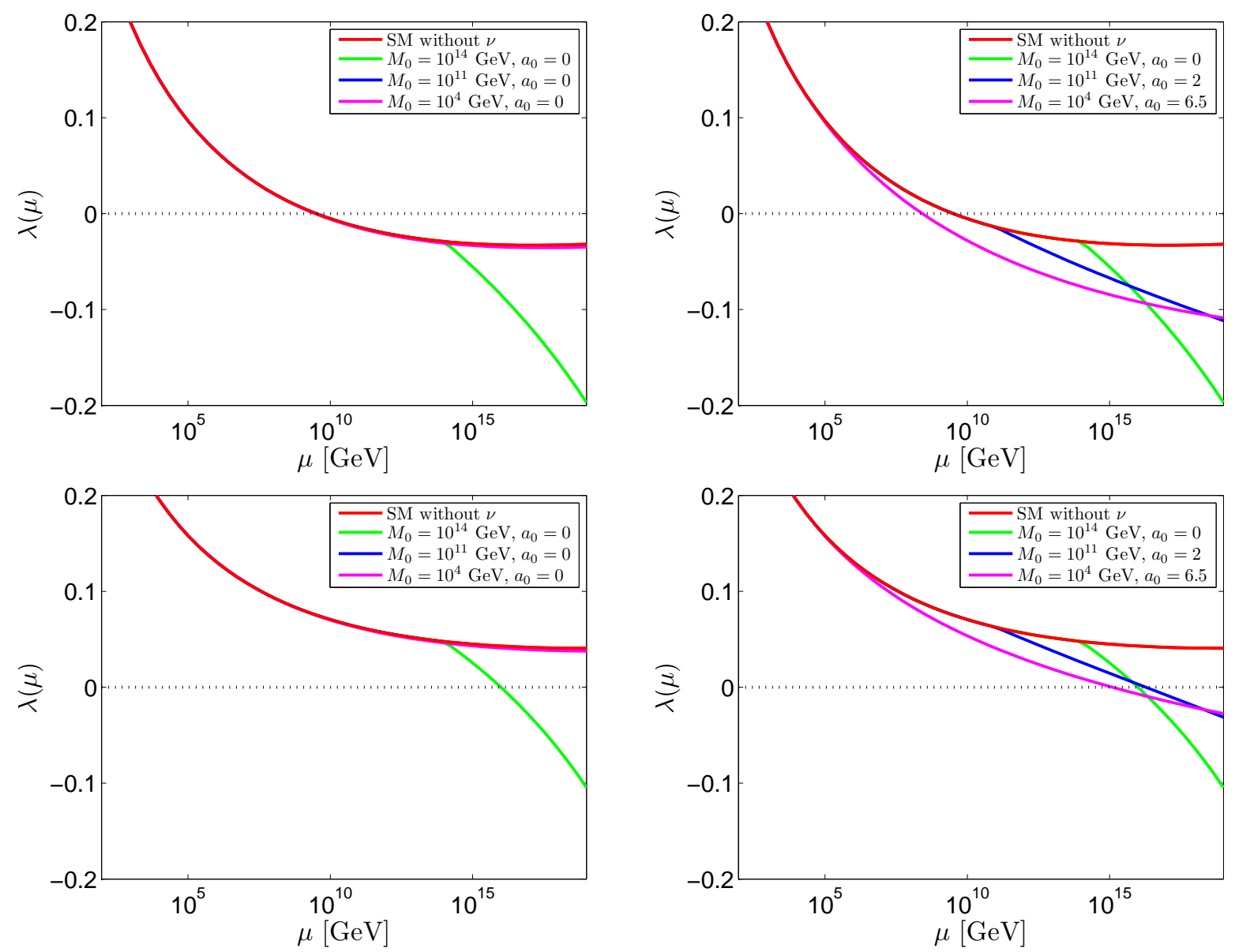

FIG. 1: RG evolution of the Higgs self-coupling for $m_{H}=125 \mathrm{GeV}$ (upper panel) and $m_{H}=$ $135 \mathrm{GeV}$ (lower panel) with $m_{1}=0.1 \mathrm{eV}$. Red curves correspond to the SM without right-handed neutrinos, while for green and blue curves we include right-handed neutrino contributions and take the right-handed neutrino thresholds $M_{0}=10^{14} \mathrm{GeV}$ (green), $M_{0}=10^{11} \mathrm{GeV}$ (blue) and $M_{0}=10 \mathrm{TeV}$ (magenta). In the left plots we switch off the $A$ matrix by setting $a_{0}=0$, whereas for the right plots, we take $a_{0}=2$ and $a_{0}=6.5$ for the green and blue curves, respectively.

\section{B. Higgs mass window and cutoff scale}

The effect of right-handed neutrinos on the stability bounds is shown in Fig. 2 for particular choices of parameters. The threshold effects take place at $\mu=M_{0}$ and can be identified easily on the plot. In the SM framework, there is essentially no stability constraint for Higgs masses larger than about $130 \mathrm{GeV}$, whereas in the presence of right-handed neutrinos the cutoff scale $\Lambda$ is decreased significantly, in particular for low scale seesaw models with large $A$ matrix elements. For completeness, we also show in Fig. 2 the triviality bounds on the Higgs mass improved by including right-handed neutrinos, i.e., $\lambda(\mu)<4 \pi$. As expected, there is very little effect from seesaw. One can read from the plot that the Higgs mass window becomes narrower when the right-handed neutrinos are added, which is mainly due 


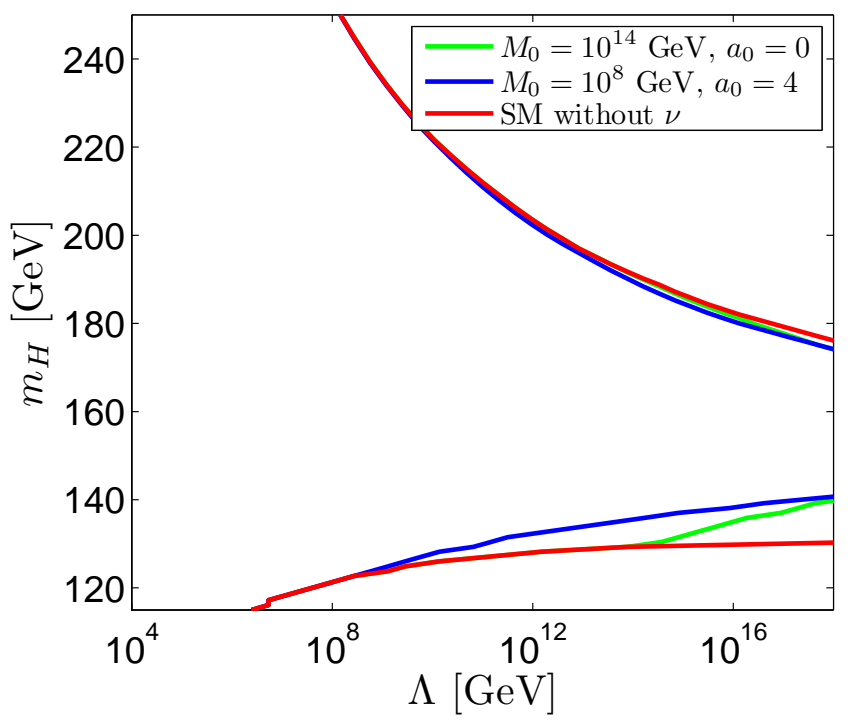

FIG. 2: Higgs mass window from stability and triviality bounds. The red curve corresponds to the case without right-handed neutrinos, while for green and blue curves we take $\left(M_{0}=10^{14} \mathrm{GeV}, a_{0}=\right.$ $0)$ and $\left(M_{0}=10^{8} \mathrm{GeV}, a_{0}=4\right)$, respectively.

to the strengthening of the stability bounds.

As mentioned above, in determining the cutoff scale of possible new physics, the neutrino Yukawas can be crucial and may affect $\beta_{\lambda}$ in a similar way as the top quark mass. We illustrate in Fig. 3 the allowed parameter ranges in the $m_{H}-M_{0}$ plane by choosing the light neutrino mass $m_{1}=0.1 \mathrm{eV}$ and $a_{0}=2$. One can read from the plot that, for a chosen Higgs mass smaller than $125 \mathrm{GeV}$, the cutoff scale is essentially not affected by right-handed neutrinos (cf. the horizontal lines at the bottom of Fig. 3i), since the corresponding Yukawa couplings are not significant enough when right-handed neutrino thresholds are lighter than $10^{10} \mathrm{GeV}$. The situation becomes more complicated for a Higgs mass in the intermediate range, i.e., $130 \mathrm{GeV} \lesssim m_{H}<180 \lesssim \mathrm{GeV}$, owing to the fact that there is essentially no stability constraint in the pure SM framework (i.e., $\Lambda$ can be as large as the Planck scale $10^{19} \mathrm{GeV}$ ). For example, if the Higgs mass is located at $m_{H}=140 \mathrm{GeV}$, the cutoff scale $\Lambda$ decreases with increasing $M_{0}$, because $Y_{\nu}$ becomes larger in this case. When $\Lambda \gtrsim M_{0}, \Lambda$ stops decreasing and achieves a minimum, which can be seen from the plot for right-handed neutrino masses around a critical scale $M_{0}^{\text {cric }} \sim 10^{12} \mathrm{GeV}$. This can be easily understood because $\Lambda$ cannot be smaller than $M_{0}$, otherwise the right-handed neutrino threshold will not be crossed. ${ }^{2}$ Then, for right-handed neutrino masses in the range $M_{0}>M_{0}^{\text {cric }}$, the RG evolution between $M_{0}$ and $\Lambda$ is strongly enhanced by larger $Y_{\nu}$, which drives $\lambda$ to zero very

\footnotetext{
${ }^{2}$ Note that a negative $\lambda$ arises for $\Lambda<M_{0}$, which, however, does not necessarily mean that the model is invalid, since the unlikely possibility of a metastable electroweak vacuum exists. For a general discussion on this topic, see, e.g. Ref. [6].
} 


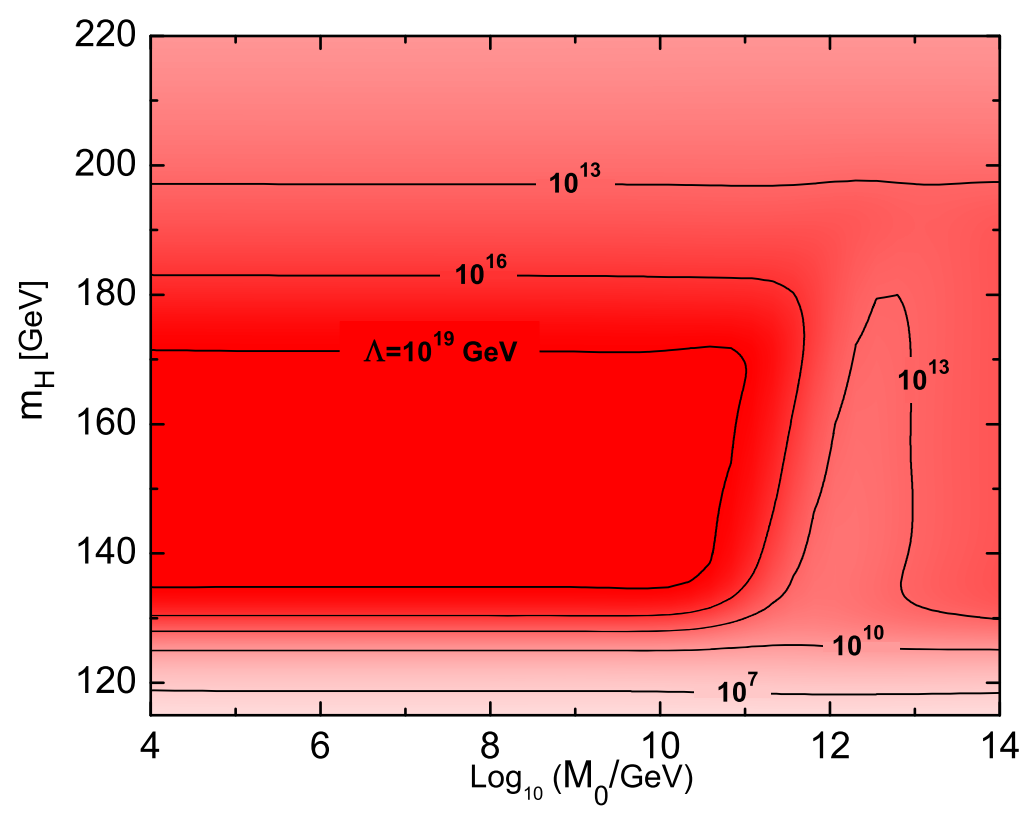

FIG. 3: Illustration for the parameter space of $m_{H}$ and $M_{0}$, for different choices of the cutoff scale $\Lambda$. Darker areas correspond to higher cutoff scales allowed by the stability and triviality boundary conditions. The other input parameters are taken as $m_{1}=0.1 \mathrm{eV}$ and $a_{0}=2$.

efficiently, even for a small energy interval. Therefore, the cutoff $\Lambda$ will remain close to $M_{0}$, which is reflected in Fig. 通 by the vertical parts of the contour $\Lambda=10^{13} \mathrm{GeV}$. Finally, when $m_{H}>180 \mathrm{GeV}, \Lambda$ is mainly constrained by the triviality bounds (cf. the horizontal lines at the top of Fig. 3). We should note that the features discussed here do not rely on the specific choice of model parameters. In general, the value of $M_{0}^{\text {cric }}$ decreases with decreasing $a_{0}$.

We also show in Fig. 4 the parameter space of $M_{0}$ and $a_{0}$ constrained by the stability bound for $m_{H}=125 \mathrm{GeV}$ and $135 \mathrm{GeV}$. From the plot one observes that the role of righthanded neutrinos becomes visible in case of a relatively large $a_{0}$ (e.g., $a_{0}>4$ ). In general, for a chosen $M_{0}$, the larger $a_{0}$ the smaller the cutoff scale one can expect. Interestingly, when both $M_{0}$ and $a_{0}$ are sizable, $\Lambda$ remains close to the right-handed neutrino masses $\left(\Lambda \simeq M_{0}\right)$, due to the same reason mentioned in the above paragraph. This feature is shown by the vertical curves.

The RG evolution of $\lambda$ depends also on the light neutrino masses as can be seen in Eq. (10). Thus, we illustrate in Fig. $[$ the dependence of $\Lambda$ on the lightest neutrino mass $m_{1}$. One can observe from the plot that, for a chosen right-handed neutrino scale, the larger the light neutrino mass, the lower the value at which $\Lambda$ could be located. This is in good agreement with our analytical results [cf. Eqs. (10) and (11)]. Moreover, $\Lambda$ is very sensitive to $a_{0}$, in particular when $a_{0}$ is large. Note however that $a_{0}$ cannot be arbitrarily large since 

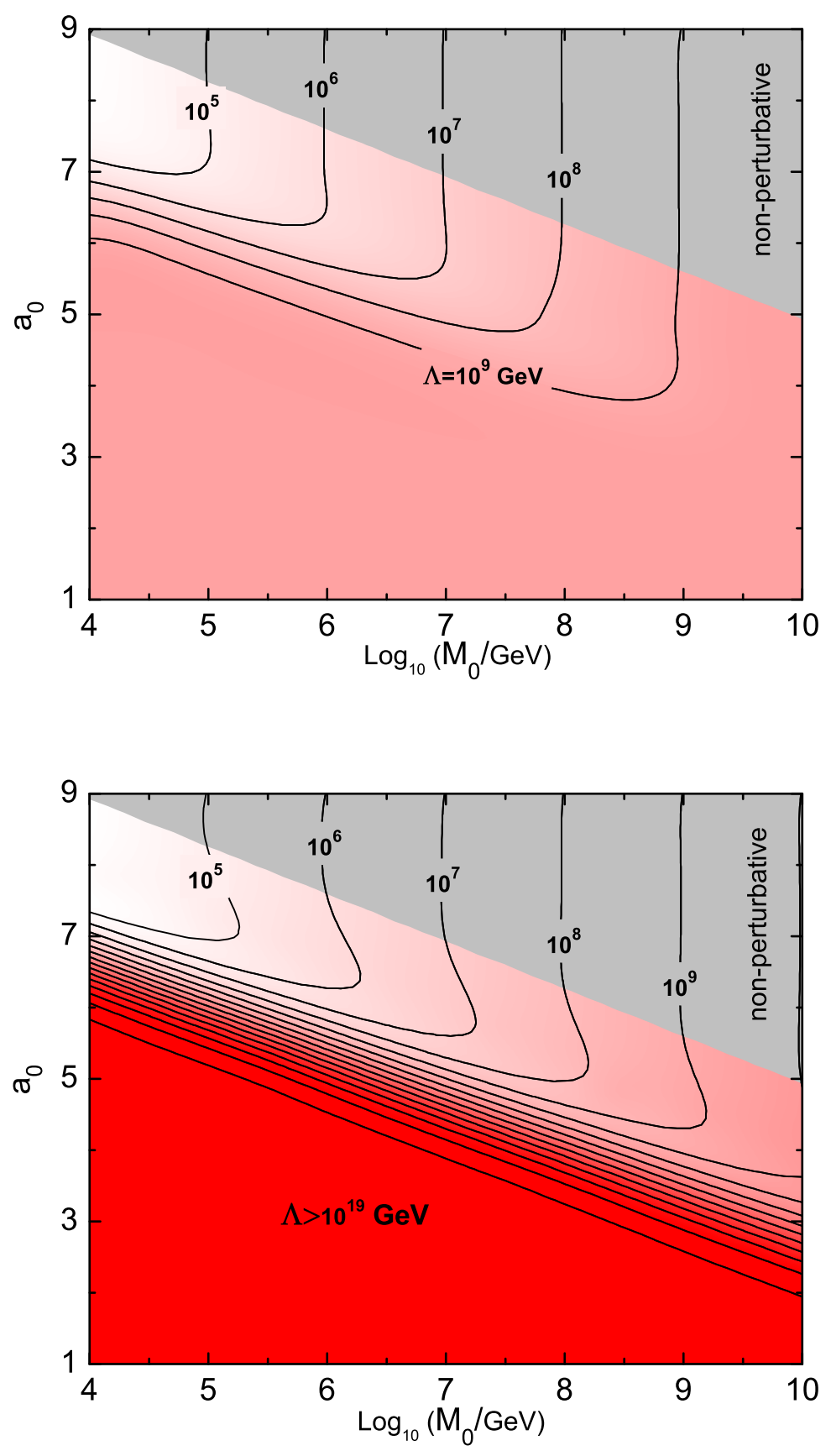

FIG. 4: Parameter ranges of $M_{0}$ and $a_{0}$ for $m_{1}=0.1 \mathrm{eV}, m_{H}=125 \mathrm{GeV}$ (upper plot) and $m_{H}=135 \mathrm{GeV}$ (lower plot). The areas shaded in gray correspond to non-perturbative couplings, i.e., the absolute value of one entry in $Y_{\nu}$ is larger than $4 \pi$.

$Y_{\nu}$ suffers from the perturbativity constraint. Furthermore, when right-handed neutrinos are relatively light (e.g. $\lesssim 10 \mathrm{TeV}$ ), the unitarity of the PMNS matrix $U$ sets constraints on the mixing between light and heavy neutrinos, suggesting roughly $\left(Y_{\nu} v / M_{0}\right)^{2} \lesssim 10^{-3}[26]$. 

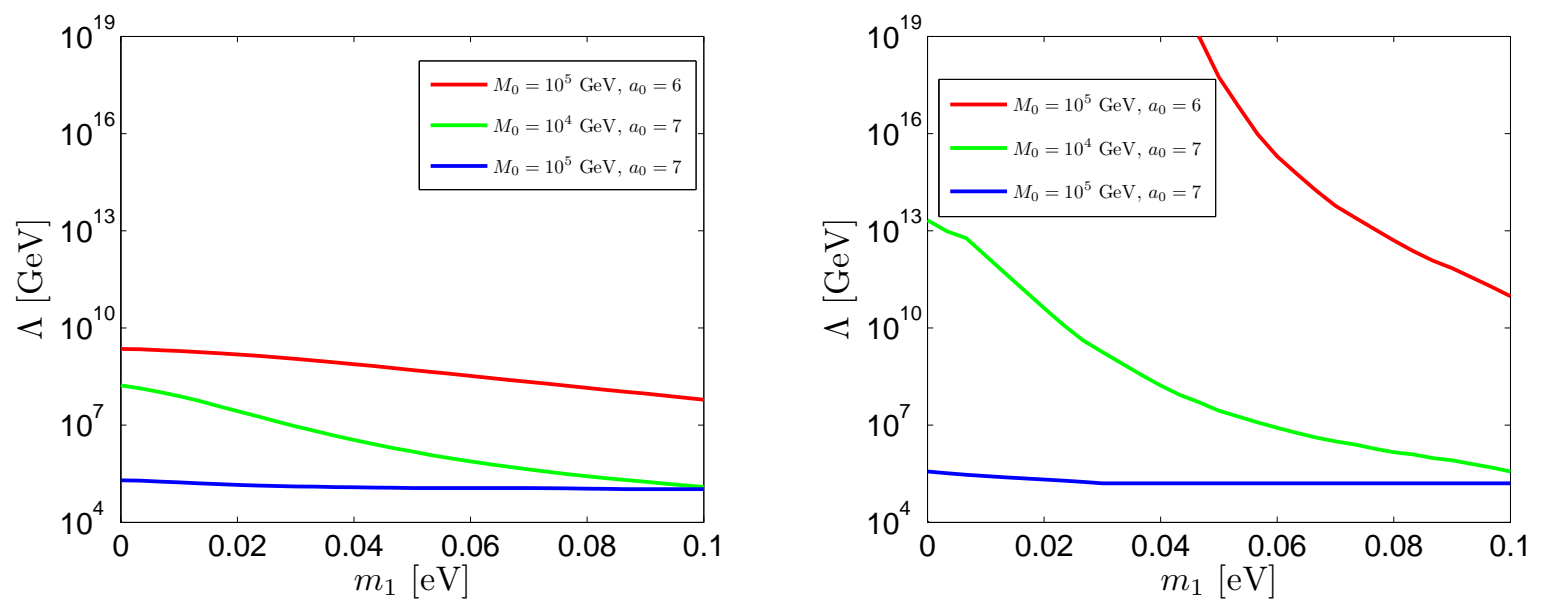

FIG. 5: Cutoff scale $\Lambda$ with respect to the lightest neutrino mass $m_{1}$ for $m_{H}=125 \mathrm{GeV}$ (left plot) and $m_{H}=135 \mathrm{GeV}$ (right plot). Red, green, and blue curves correspond to the parameter choices $\left(M_{0}=10^{5} \mathrm{GeV}, a_{0}=6\right),\left(M_{0}=10^{4} \mathrm{GeV}, a_{0}=7\right)$ and $\left(M_{0}=10^{5} \mathrm{GeV}, a_{0}=7\right)$, respectively.

\section{RG evolution of $\lambda$ in realistic low scale seesaw models}

While the discussion made so far used a general parametrization of the unknown neutrino Yukawas, specific models or frameworks constructed in order to predict certain features of lepton mixing will be able to provide more definite information on the flavor structure. We will shortly discuss two examples. Both of them were constructed in order to have sizable cross sections for the production of heavy neutrinos at the LHC.

Model A: We first consider an $A_{4}$ type I seesaw model described in Ref. [18], in which right-handed neutrinos are assigned to the three-dimensional representation of $A_{4}$ while lepton doublets transform under $\mathbf{1}^{\prime \prime}$. At leading order, $Y_{\nu}$ is given by

$$
Y_{\nu}=\left(\begin{array}{ccc}
h_{1} & h_{2} & h_{3} \\
\omega h_{1} & \omega h_{2} & \omega h_{3} \\
\omega^{2} h_{1} & \omega^{2} h_{2} & \omega^{2} h_{3}
\end{array}\right)
$$

with $\omega=e^{i \frac{2}{3} \pi}$, and the right-handed neutrino mass matrix is proportional to a unit matrix, i.e., $M_{R}=M_{0} \mathbb{1}$. Using the seesaw formula (5), one can easily see that light neutrinos are massless in the leading order approximation. Small perturbations or explicit symmetry breaking terms are therefore needed to include neutrino masses. We can ignore them for the purpose of our study. The neutrino contribution to $\beta_{\lambda}$ is estimated by

$$
\operatorname{tr}\left(Y_{\nu}^{\dagger} Y_{\nu}\right) \simeq 3\left(h_{1}^{2}+h_{2}^{2}+h_{3}^{2}\right)
$$



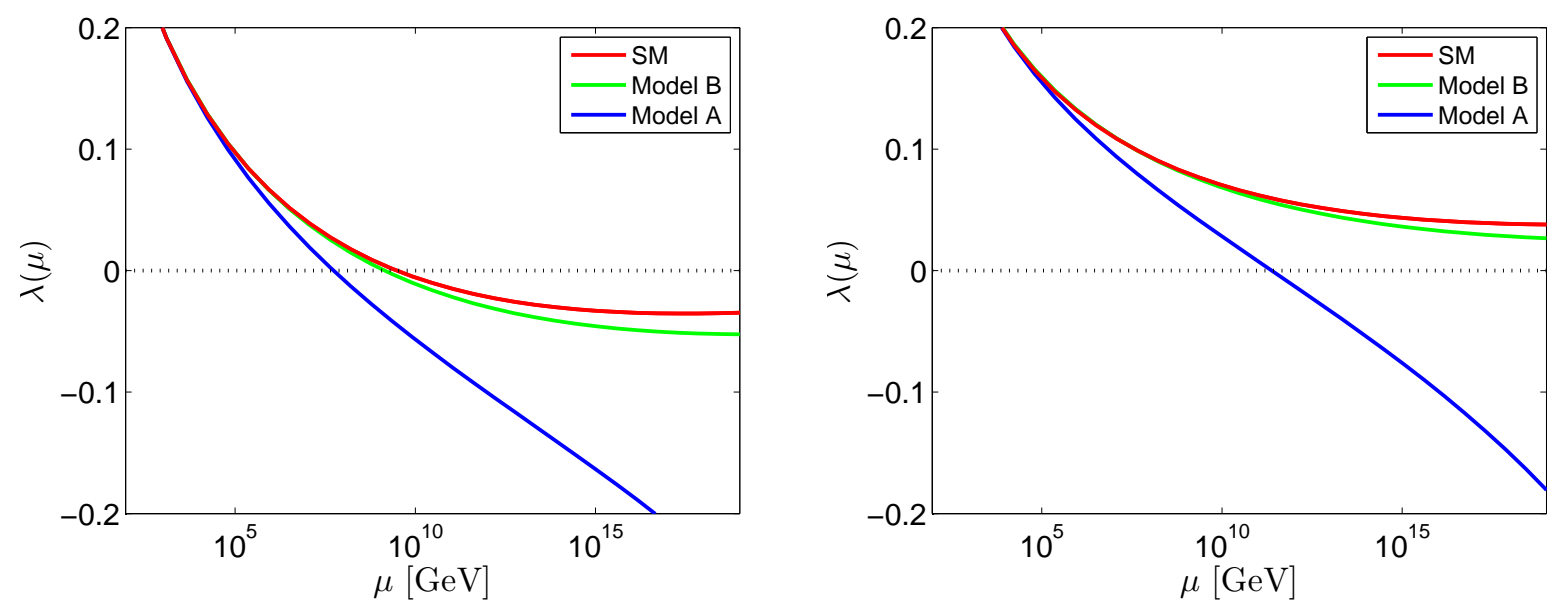

FIG. 6: RG evolution of the Higgs self-coupling for $m_{H}=125 \mathrm{GeV}$ (left plot) and $m_{H}=135 \mathrm{GeV}$ (right plot). Red curves correspond to the simplest SM without right-handed neutrinos, while for the green (model A) and blue (model B) curves, we take the right-handed neutrino threshold $M_{0}=350 \mathrm{GeV}$. The Yukawa coupling parameters are chosen to be $h_{1}=h_{2}=h_{3}=0.15$ and $y_{1}=y_{2}=y_{3}=0.15$, respectively.

As an example, we take $h_{1}=h_{2}=h_{3}=0.15$ together with $M_{0}=350 \mathrm{GeV}$, for which a discovery search can be performed at the LHC [25]. We illustrate in Fig. 6] the effect of right-handed neutrinos on the RG evolution of $\lambda$. Similar to Fig. 1, the right-handed neutrino corrections are noteworthy compared to the SM evolution.

Model B: Next we consider the minimal TeV seesaw model [19], in which only two righthanded neutrinos are introduced, having opposite CP parity. In this model, right-handed neutrinos can be paired together to form a pseudo-Dirac fermion, and light neutrinos are massless at leading order due to the conservation of lepton number. Effectively, at leading order, the Yukawa coupling matrix is given by

$$
Y_{\nu}=\left(\begin{array}{ccc}
y_{1} & y_{2} & y_{3} \\
0 & 0 & 0
\end{array}\right)
$$

while the right-handed mass matrix takes a $2 \times 2$ form,

$$
M_{R}=\left(\begin{array}{cc}
0 & M_{0} \\
M_{0} & 0
\end{array}\right) \text {. }
$$




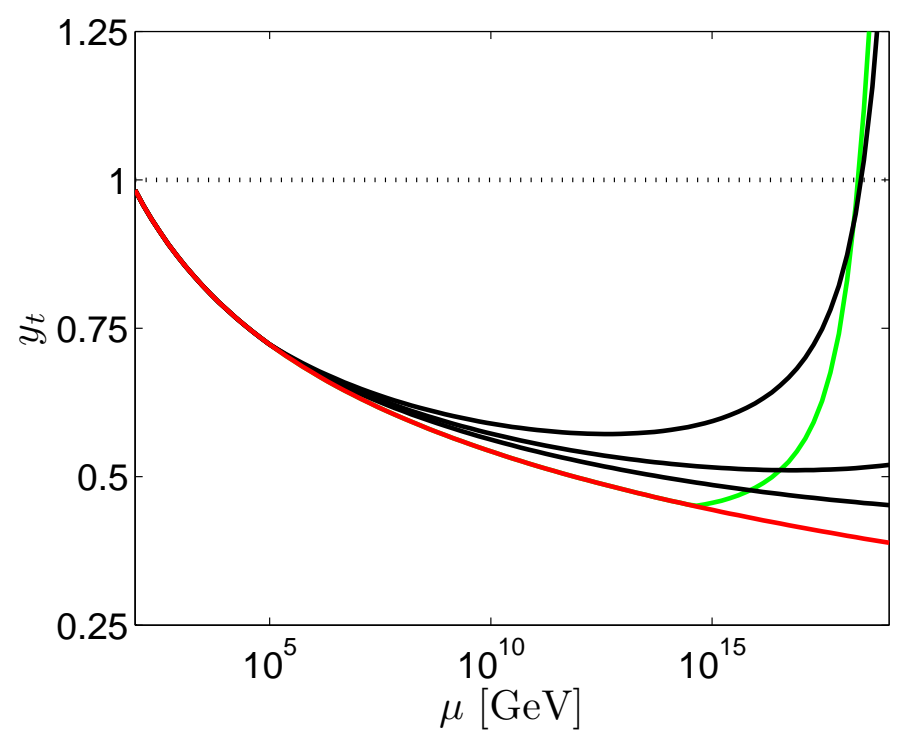

FIG. 7: RG evolution of top quark Yukawa coupling $y_{t}$. We take the input parameters $m_{H}=$ $125 \mathrm{GeV}$ and $m_{1}=0.1 \mathrm{eV}$. The red curve corresponds to the pure SM case without right-handed neutrinos, while the green curve is for $M_{0}=4 \times 10^{14} \mathrm{GeV}$ and $a_{0}=0$. The black curves correspond to $M_{0}=10^{5} \mathrm{GeV}$ with $a_{0}=6.0,6.1,6.2$ from bottom to top.

It is not difficult to calculate that ${ }^{3}$

$$
\operatorname{tr}\left(Y_{\nu}^{\dagger} Y_{\nu}\right) \simeq y_{1}^{2}+y_{2}^{2}+y_{3}^{2}
$$

The running of $\lambda$ for the parameter choice $y_{1}=y_{2}=y_{3}=0.15$ is shown in Fig. 6. In comparison to model $\mathrm{A}$, a factor 3 is missing in $\operatorname{tr}\left(Y_{\nu}^{\dagger} Y_{\nu}\right)$, and therefore the modification to the running of $\lambda$ is milder.

Note that, in order to accommodate non-vanishing neutrino masses in those models, small lepton number violating perturbations have to be introduced [18-20], whose stability has to be guaranteed by symmetries. Explicitly, in order to generate sub-eV light neutrino masses, one needs perturbation terms $\epsilon$ in the Yukawa matrix of the order $\epsilon \sim \frac{m_{\nu} M_{R}}{y_{i} v^{2}}$. Taking Model $\mathrm{B}$ as an example, we can estimate that $\epsilon \sim 10^{-11}$. The mechanism behind this stability and the precise values of the parameters do not affect the main results of this work.

\footnotetext{
${ }^{3}$ Note that diagonalization of $M_{R}$ is not necessary, because the unitary matrix $V$ that diagonalizes it will appear together with $Y_{\nu}$ as $V Y_{\nu}$ and thus drops out of $Y_{\nu}^{\dagger} Y_{\nu}$.
} 


\section{Top quark Yukawa coupling}

The Dirac Yukawas influence all $\beta$-functions of the SM, in particular the one of the top quark Yukawa coupling $y_{t}$. Its $\beta$-function approximately reads [29]

$$
\frac{\mathrm{d} y_{t}}{\mathrm{~d} \ln \mu} \equiv \beta_{y_{t}} \simeq \frac{11}{3} y_{t}^{3}+\operatorname{tr}\left(Y_{\nu}^{\dagger} Y_{\nu}\right) y_{t}-\left(\frac{17}{20} g_{1}^{2}+\frac{9}{4} g_{2}^{2}+8 g_{3}^{2}\right) y_{t}
$$

at energy scales above the right-handed neutrino threshold. Recall that, in the SM, the top quark Yukawa coupling tends to be smaller at higher energy scale due to the negative contribution of gauge couplings in $\beta_{y_{t}}$ (see e.g., [28]). Since the neutrino Yukawas contribute with a positive sign, the running behavior of $y_{t}$ may be significantly modified. The RG evolution of $y_{t}$ is depicted in Fig. 7 for some examples. An interesting observation is that the exact value $y_{t}=1$ (which is impossible in the SM) could be obtained at higher energy scales, indicating the restoration of certain kinds of Yukawa unifications or flavor symmetries. We see that this value can be reached for the case of low scale seesaw with non-trivial flavor structure as well as for the canonical case with $M_{0}$ being of order $10^{14} \mathrm{GeV}$.

\section{CONCLUSIONS}

We have considered here the influence of neutrino Yukawa couplings in the type I seesaw mechanism on the Higgs self-coupling. The impact is similar to the one of the up-quarks, and thus can modify the vacuum stability bounds at low Higgs masses. While naively this effect decreases with decreasing seesaw scale, we have noted here that this is not necessarily the case when the flavor structure of the matrices is taken into account. Providing a general analysis with a parametrization of the Yukawas, as well as giving two model examples, we showed their impact. The Higgs mass window can become narrower and the cutoff scale could become lower than in the Standard Model case. It is worth to remark that the instability bound should not be viewed as a severe problem of the model since the SM is likely to be embedded in a more fundamental framework, and the actual fate of the electroweak vacuum depends on the cosmological history. In some other extensions of the SM, the interactions between new particles and the SM Higgs coublet may lead to a positive contribution to the Higgs quartic coupling, which could stabilize the Higgs potential (see, e.g. discussions in Ref. [32]). Nevertheless, whenever the seesaw parameters are such that production of righthanded neutrinos at colliders is possible, the effects discussed in this work can be expected. In addition, the neutrino Yukawas may have an effect also on the evolution of other Standard Model parameters, which we illustrated with the example of the top quark Yukawa reaching exactly the value 1 at high scale.

In general, seesaw contributions to the Higgs self-coupling are a particularly simple and

natural example on the impact of new physics on fundamental properties of the Standard 
Model.

\section{Acknowledgments}

We are grateful to Manfred Lindner and Martin Holthausen for useful comments. This work was supported by the ERC under the Starting Grant MANITOP.

[1] G. Aad et al. (ATLAS Collaboration) (2012), 1202.1408.

[2] S. Chatrchyan et al. (CMS Collaboration) (2012), 1202.1488.

[3] W. Fisher (Talk presented at Rencontres de Moriond EW 2012, La Thuile, Italy, 2012), http://indico.in2p3.fr/getFile.py/access?contribId=86\&sessionId=1\&resId=0\& materialId=slides\&conf $I d=6001$.

[4] T. Aaltonen et al. (ALEPH Collaboration, CDF Collaboration, D0 Collaboration, DELPHI Collaboration, L3 Collaboration, OPAL Collaboration, SLD Collaboration, LEP Electroweak Working Group, Tevatron Electroweak Working Group, SLD Electroweak and Heavy Flavour Groups) (2010), 1012.2367.

[5] A. D. Linde, JETP Lett. 23, 64 (1976); A. D. Linde, Phys.Lett. B62, 435 (1976); S. Weinberg, Phys.Rev.Lett. 36, 294 (1976); H. Politzer and S. Wolfram, Phys.Lett. B82, 242 (1979); P. Q. Hung, Phys.Rev.Lett. 42, 873 (1979); N. Cabibbo, L. Maiani, G. Parisi, and R. Petronzio, Nucl.Phys. B158, 295 (1979); R. A. Flores and M. Sher, Phys.Rev. D27, 1679 (1983); M. Lindner, Z.Phys. C31, 295 (1986).

[6] M. Sher, Phys. Rept. 179, 273 (1989).

[7] M. Lindner, M. Sher, and H. W. Zaglauer, Phys.Lett. B228, 139 (1989); J. Casas, J. Espinosa, and M. Quiros, Phys.Lett. B342, 171 (1995), hep-ph/9409458.

[8] J. F. Gunion, H. E. Haber, G. L. Kane, and S. Dawson, Front.Phys. 80, 1 (2000); S. Dawson (1998), hep-ph/9901280.

[9] M. Holthausen, K. S. Lim, and M. Lindner, JHEP 1202, 037 (2012), 1112.2415.

[10] J. Elias-Miro, J. R. Espinosa, G. F. Giudice, G. Isidori, A. Riotto, et al., Phys.Lett. B709, $222(2012), 1112.3022$.

[11] Z.-z. Xing, H. Zhang, and S. Zhou (2011), 1112.3112.

[12] P. Minkowski, Phys. Lett. B67, 421 (1977); T. Yanagida, in Proc. Workshop on the baryon number of the Universe and unified theories, edited by O. Sawada and A. Sugamoto (1979), p. 95; R. N. Mohapatra and G. Senjanović, Phys. Rev. Lett. 44, 912 (1980); M. Gell-Mann, P. Ramond, and R. Slansky, in Supergravity, edited by P. van Nieuwenhuizen and D. Freedman (1979), p. 315.

[13] B. Grzadkowski and M. Lindner, Phys.Lett. B193, 71 (1987); Y. Pirogov and O. Zenin, Eur.Phys.J. C10, 629 (1999), hep-ph/9808396. 
[14] J. Casas, V. Di Clemente, A. Ibarra, and M. Quiros, Phys.Rev. D62, 053005 (2000), hep$\mathrm{ph} / 9904295$.

[15] I. Gogoladze, N. Okada, and Q. Shafi, Phys.Lett. B668, 121 (2008), 0805.2129.

[16] B. He, N. Okada, and Q. Shafi, Phys.Lett. B695, 219 (2011), 1009.1878.

[17] C.-S. Chen and Y. Tang (2012), 1202.5717.

[18] J. Kersten and A. Y. Smirnov, Phys. Rev. D76, 073005 (2007), 0705.3221.

[19] M. Gavela, T. Hambye, D. Hernandez, and P. Hernandez, JHEP 0909, 038 (2009), 0906.1461;

H. Zhang and S. Zhou, Phys.Lett. B685, 297 (2010), 0912.2661.

[20] R. Adhikari and A. Raychaudhuri, Phys.Rev. D84, 033002 (2011), 1004.5111; T. Ohlsson, C. Popa, and H. Zhang, Phys.Lett. B692, 257 (2010), 1007.0106; A. Ibarra, E. Molinaro, and S. Petcov, Phys.Rev. D84, 013005 (2011), 1103.6217; N. Haba, T. Horita, K. Kaneta, and Y. Mimura (2011), 1110.2252.

[21] J. Casas and A. Ibarra, Nucl.Phys. B618, 171 (2001), hep-ph/0103065.

[22] S. Pascoli, S. Petcov, and C. Yaguna, Phys.Lett. B564, 241 (2003), hep-ph/0301095.

[23] A. Datta, M. Guchait, and A. Pilaftsis, Phys. Rev. D50, 3195 (1994), hep-ph/9311257.

[24] T. Han and B. Zhang, Phys. Rev. Lett. 97, 171804 (2006), hep-ph/0604064.

[25] F. del Aguila, J. A. Aguilar-Saavedra, and R. Pittau, JHEP 10, 047 (2007), hep-ph/0703261.

[26] S. Bergmann and A. Kagan, Nucl.Phys. B538, 368 (1999), hep-ph/9803305; B. Bekman, J. Gluza, J. Holeczek, J. Syska, and M. Zralek, Phys.Rev. D66, 093004 (2002), hep$\mathrm{ph} / 0207015$.

[27] M. Fukugita and T. Yanagida, Phys. Lett. B174, 45 (1986).

[28] Z.-z. Xing, H. Zhang, and S. Zhou, Phys. Rev. D77, 113016 (2008), 0712.1419.

[29] M. E. Machacek and M. T. Vaughn, Nucl.Phys. B222, 83 (1983); M. E. Machacek and M. T. Vaughn, Nucl.Phys. B236, 221 (1984); M. E. Machacek and M. T. Vaughn, Nucl.Phys. B249, 70 (1985); H. Arason, D. Castano, B. Keszthelyi, S. Mikaelian, E. Piard, et al., Phys.Rev. D46, 3945 (1992); C. Ford, D. Jones, P. Stephenson, and M. Einhorn, Nucl.Phys. B395, 17 (1993), hep-lat/9210033; M.-x. Luo and Y. Xiao, Phys.Rev.Lett. 90, 011601 (2003), hep-ph/0207271.

[30] A. Sirlin and R. Zucchini, Nucl.Phys. B266, 389 (1986); T. Hambye and K. Riesselmann, Phys.Rev. D55, 7255 (1997), hep-ph/9610272.

[31] K. Nakamura et al. (Particle Data Group), J.Phys.G G37, 075021 (2010).

[32] J. Elias-Miro, J. R. Espinosa, G. F. Giudice, H. M. Lee, and A. Strumia (2012), 1203.0237. 